\section{A saúde do adolescente em tempos da COVID-19: scoping review}

\author{
Adolescents' health in times of COVID-19: \\ a scoping review
}

La salud del adolescente en tiempos de la COVID-19: scoping review

\section{Resumo}

A COVID-19, doença provocada pelo SARS-CoV-2 (novo coronavírus), surgiu na China em dezembro de 2019 e se espalhou rapidamente por todo o mundo. Diante desse cenário, este estudo objetivou identificar o impacto ou os efeitos da pandemia da COVID-19 na saúde dos adolescentes. Trata-se de uma revisão da literatura do tipo scoping review valendo-se das seguintes bases de dados: Web of Science; CINAHL; PsycINFO; SciELO; PUBCOVID19. O estudo seguiu as etapas propostas pelo Instituto Joanna Briggs para scoping reviews, e a questão que orientou os procedimentos adotados foi construída pelo acrônimo PCC (população; conceito; contexto). Foram incluídos 11 artigos na revisão. Clinicamente, os adolescentes apresentam os mesmos sintomas da COVID-19 que os adultos acometidos pela doença. Verificou-se que a pandemia e as medidas sanitárias adotadas para controlar a contaminação são associadas a problemas de saúde mental em adolescentes. Especificamente, os adolescentes têm vivenciado de forma negativa as medidas de distanciamento social e fechamento das escolas. Essas medidas também podem favorecer a ocorrência de violências ou comportamentos agressivos no contexto doméstico. Serviços de saúde que atendem a população adolescente precisaram reorientar as práticas de cuidado, adotando o modelo virtual em substituição ao cuidado presencial, e mesmo as pesquisas precisaram ser repensadas. Esta scoping review abordou um tema emergente em relação a uma população pouco considerada nos estudos sobre a COVID-19. Os resultados sugerem que a situação de pandemia pode ser considerada um determinante que afeta diferentes dimensões da vida dos adolescentes.

COVID-19; Pandemias; Quarentena; Saúde do Adolescente; Vulnerabilidade em Saúde
Wanderlei Abadio de Oliveira 1

Jorge Luiz da Silva 2

André Luiz Monezi Andrade 1

Denise De Micheli 3

Diene Monique Carlos 4

Marta Angélica Iossi Silva 5

doi: 10.1590/0102-311X00150020

\author{
Correspondência \\ W. A. Oliveira \\ Programa de Pós-graduação em Psicologia, Pontifícia \\ Universidade Católica de Campinas. \\ Av. John Boyd Dunlop s/n, Campinas, SP 13060-904, Brasil. \\ wanderleio@hotmail.com

\footnotetext{
1 Programa de Pós-graduação em Psicologia, Pontifícia Universidade Católica de Campinas, Campinas, Brasil. 2 Programa de Pós-graduação em Promoção da Saúde, Universidade de Franca, Franca, Brasil.

3 Departamento de Psicobiologia, Universidade Federal de São Paulo, São Paulo, Brasil.

4 Departamento de Enfermagem, Universidade Federal de São Carlos, São Carlos, Brasil.

5 Escola de Enfermagem de Ribeirão Preto, Universidade de São Paulo, Ribeirão Preto, Brasil.
} 


\section{Introdução}

A adolescência é um período do desenvolvimento marcado por transformações biológicas, psicológicas e sociais. As características desse momento são complexas, e múltiplas abordagens procuram responder como qualificar esse período do ciclo vital situado entre a infância e a idade adulta. Uma das formas de delimitar a adolescência é a partir do marco etário. Nesse sentido, uma discussão atual entre pesquisadores europeus e australianos sugere que a definição cronológica da adolescência seja ampliada, comparando com demarcações de décadas passadas e compreendendo, atualmente, o período entre 10 e 24 anos 1. Essa ampliação etária tende a corrigir distorções nas concepções anteriores, incluindo as mudanças sociais e a consequente necessidade de investimentos prolongados no que se refere aos cuidados em saúde dessa população.

Esses aspectos influenciam as políticas e programas de saúde do adolescente e, no caso do Brasil, podem ser exemplificados pelo Programa Saúde na Escola, instituído em 2007, e pelas Diretrizes Nacionais para a Atenção Integral à Saúde de Adolescentes e Jovens na Promoção, Proteção e Recuperação da Saúde de $2010^{2}$. As intervenções decorrentes desses documentos enfatizam a importância de não se considerar o adolescente e o processo de adolescer como problemas, mas como uma janela de oportunidades de um sujeito em desenvolvimento inserido nos contextos social, cultural e coletivo 2 . Os temas mais explorados junto aos adolescentes dizem respeito à saúde mental, à saúde sexual e reprodutiva, à saúde bucal e violências em iniciativas de promoção ou educação em saúde 2 . Segundo a literatura científica, as intervenções junto a esse público devem responder às necessidades dos adolescentes que são os protagonistas das iniciativas 3 .

Atualmente, a COVID-19, doença provocada pelo SARS-CoV-2 que surgiu no final de $2019 \mathrm{em}$ Wuhan, na China, foi declarada pela Organização Mundial da Saúde (OMS) como a sexta emergência de saúde pública que exige atenção, sendo designada como pandêmica 4 . As medidas sanitárias de controle do contágio para garantir a proteção da população, desacelerar a tendência crescente de transmissão e impedir o colapso dos serviços de saúde se configuram como elementos que alteram o cotidiano da vida das pessoas 5,6. Especificamente, segundo a OMS, o fechamento das instituições de ensino como iniciativa para a contenção de casos da COVID-19 retirou cerca de 1,5 bilhão de crianças e adolescentes das escolas 7 . Escolas fechadas, exames e provas adiados, suspensão da conclusão de ciclos ou períodos escolares, causam uma interrupção nas rotinas e o confinamento em casa pode gerar nos adolescentes medos, incertezas, ansiedades, distanciamento social dos pares ou amigos, aspectos que afetam o bem-estar e a qualidade de vida, além de aumentar a vulnerabilidade para sofrerem diferentes tipos de violência em suas casas 8 .

Embora a COVID-19 atinja pessoas de todas as idades, nível socioeconômico, sexo e etnia, a vulnerabilidade a esta patologia está intimamente relacionada aos determinantes sociais do processo saúde/doença. Essa vulnerabilidade aumenta dependendo das condições de vida, instabilidade financeira e falta de acesso aos serviços essenciais, como saúde, educação e proteção social 9 . Nesse sentido, muitos adolescentes se encontram em condição de alta vulnerabilidade, sobretudo nas periferias das grandes cidades. No momento em que se enfrenta uma nova doença e pandemia deve-se considerar as percepções, os sentidos atribuídos à doença, saúde e risco, a cultura, o acesso à prevenção, cuidados e tratamento, aspectos presentes na abordagem dos adolescentes 9 . Diante desse cenário, o objetivo deste estudo foi realizar uma scoping review para identificar o impacto ou os efeitos da pandemia da COVID-19 na saúde do adolescente.

\section{Método}

\section{Tipo de estudo}

Trata-se de uma revisão sistemática de literatura, do tipo scoping review. De forma exploratória, uma scoping review objetiva identificar e sintetizar evidências científicas sobre questões emergentes, configurando-se como exercício preliminar à realização de uma revisão sistemática ${ }^{10}$. Seguiu-se um protocolo adequado a cada base consultada para identificar estudos elegíveis. No caso deste estudo, esse 
modelo de revisão é válido, pois ainda não estão claras as questões especificas relacionadas à saúde do adolescente e à recente pandemia da COVID-19.

\section{Procedimentos metodológicos}

Foram adotados os seguintes passos propostos pelo Instituto Joanna Briggs para scoping reviews: desenvolvimento de título, objetivo e questão norteadora; definição de critérios de inclusão e exclusão dos estudos; seleção das fontes de dados; coleta e organização dos resultados; e apresentação da revisão ${ }^{10}$. Observa-se que o título foi revisto e adequado na conclusão do estudo. Os procedimentos de revisão foram realizados por dois pesquisadores independentes que se reuniram para alcançar consensos em casos de discordância sobre a inclusão ou exclusão de artigos na revisão. A pesquisa foi desenvolvida em maio de 2020.

\section{Questão norteadora}

É a questão de pesquisa que orienta todos os procedimentos adotados na scoping review. Nesse sentido, seguiu-se o acrônimo PCC ( $\mathrm{P}=$ população; $\mathrm{C}=$ conceito; $\mathrm{C}=$ contexto) 10 para definir a seguinte pergunta orientadora: "Qual o impacto ou efeito da pandemia da COVID-19 na saúde do adolescente?”.

\section{Critérios de inclusão e exclusão}

No que se refere à população, foram incluídos estudos sobre adolescentes ou que registrem dados sobre este público. Para tanto, foi usado o marco etário proposto por pesquisadores do Centro de Saúde do Adolescente (Melbourne, Austrália), que recomenda a faixa etária da adolescência entre 10 e 24 anos 1 . O conceito central ou fenômeno de interesse examinado previu a seleção de estudos sobre os impactos e efeitos no contexto da pandemia da COVID-19. Também foram critérios de inclusão: artigos empíricos qualitativos e quantitativos; resultados de revisões de literatura; estudos teórico-reflexivos; publicados em português, inglês ou espanhol. Foram excluídos estudos com foco em outros momentos do ciclo vital (crianças ou idosos, por exemplo); cartas aos editores, pontos de vista. Não foi definido recorte temporal, a priori. Contudo, dada a atualidade do contexto investigado, apenas estudos de 2020 foram considerados.

\section{Seleção das fontes de dados}

Foram selecionadas para consulta as bases de dados Web of Science, CINAHL, PsycINFO, SciELO e na plataforma PUBCOVID19, que reúne artigos publicados sobre COVID-19 e indexados no PubMed e EMBASE. A seleção dessas bases se justifica pelo alcance multidisciplinar e ao mesmo tempo a especificidade de algumas áreas, como a saúde, por exemplo.

\section{Coleta e organização dos dados}

Inicialmente, para o reconhecimento dos descritores ou palavras-chave relacionadas à COVID-19, realizou-se uma pesquisa não sistemática nas fontes PUBCOVID19 e Web of Science. Baseando-se nesse exercício, verificou-se que as palavras mais empregadas para a indexação dos estudos sobre o contexto da pandemia atual eram: "COVID-2019", "SARS-CoV-2", "coronavirus" e "severe acute respiratory syndrome coronavirus-2". Para delimitar a população de interesse, foram incluídos os seguintes termos: "adolescence" e "adolescent". Assim como foram incluídos os termos "health" e "mental health" nos cruzamentos para contemplar os possíveis impactos da pandemia da COVID-19 nessas dimensões. Para a combinação dos termos no processo de busca foram considerados os termos booleanos AND e OR. Na SciELO os termos também foram usados em português. Por exemplo, na Web of Science foram realizados os seguintes cruzamentos: "coronavirus" OR "severe acute respiratory syndrome coronavirus-2" AND “adolescence" OR “adolescent” AND "health” OR “mental health”. Observa-se que na plataforma PUBCOVID19 os artigos são organizados em áreas temáticas e apenas as áreas "criança” e "saúde mental”' retornaram resultados sobre adolescentes. 
Foram seguidas as diretrizes do PRISMA (Preferred Reporting Items for Systematic Reviews and Meta-Analyses) para sistematizar o processo de inclusão dos estudos 11. O fluxo desse processo está apresentado na Figura 1, seguindo o modelo proposto pelo Instituto Joanna Briggs para scoping reviews.

Para a extração dos dados incluídos na revisão, dois pesquisadores independentes utilizaram uma planilha do Excel (https://www.microsoft.com) para o preenchimento das seguintes informações: autores, periódico, ano de publicação, país onde o estudo foi desenvolvido, objetivo do estudo, população/amostra, delineamento metodológico e principais resultados. Um estudo publicado em francês foi excluído. Também foram excluídos na primeira etapa de seleção cartas aos editores ou pontos de vista que eram a maioria das publicações. Não foram identificadas informações sobre o financiamento dos estudos revisados, o que impossibilitou avaliações neste aspecto. Os resultados revisados serão apresentados em tabela e no formato narrativo sobre aspectos bibliométricos e que respondem à questão que orientou a scoping review.

\section{Figura 1}

Diagrama do fluxo do processo da scoping review (PRISMA).

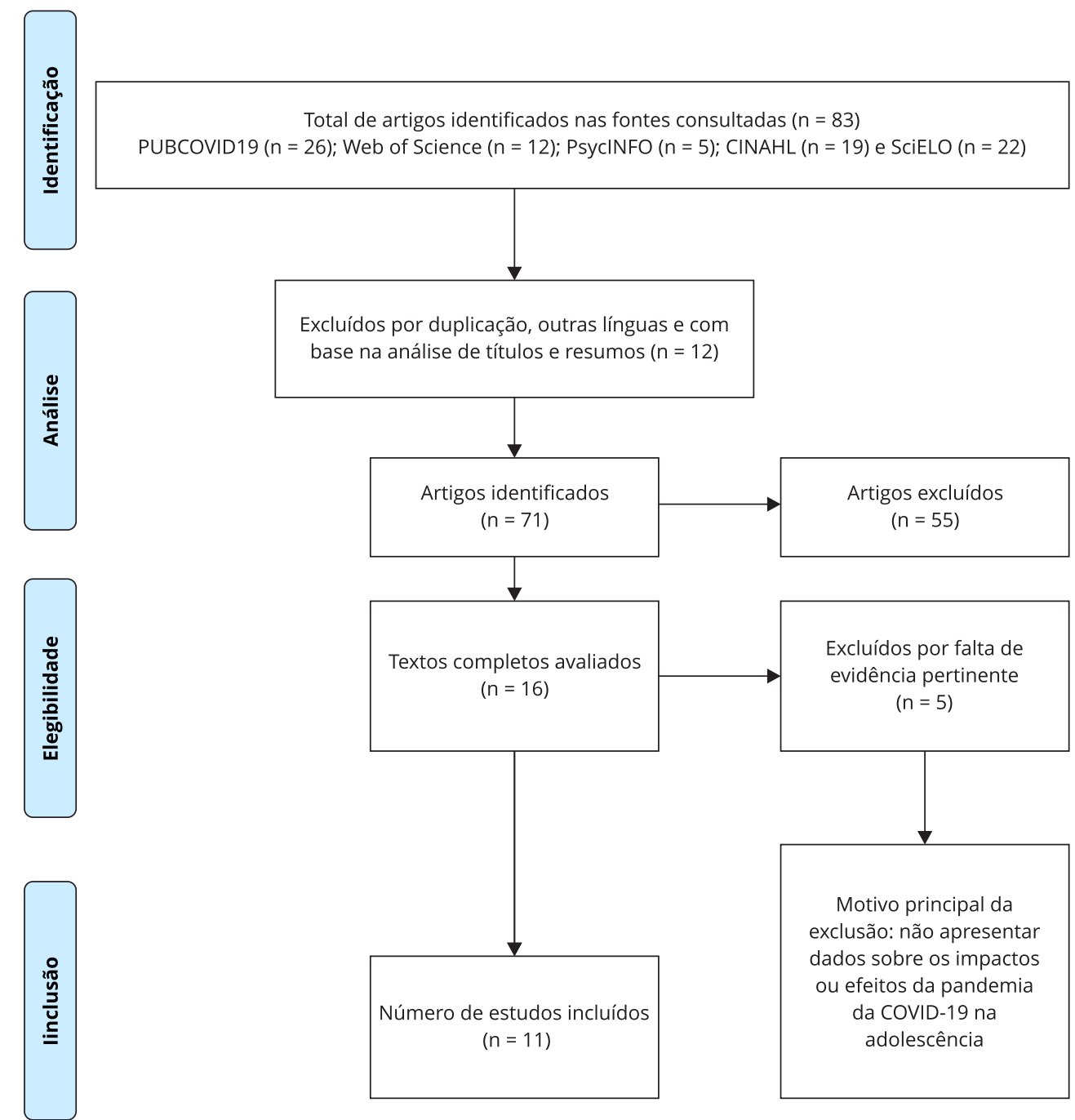




\section{Aspectos éticos}

Estudos de revisão da literatura não necessitam de aprovação em comitês de ética em pesquisa. Contudo, é importante que esse tipo de pesquisa seja fiel aos dados dos estudos originais, bem como referencie todos os textos consultados e se aplique rigor científico no tratamento dos dados. Esses aspectos éticos foram assegurados no estudo em tela.

\section{Resultados}

Após o processo de avaliação e seleção foram incluídos nesta scoping review 11 artigos científicos. O baixo número de artigos identificados na pesquisa inicial e os incluídos no estudo podem ser justificados pela atualidade e a pouca divulgação científica de pesquisas com foco na adolescência, o que ainda é uma lacuna, considerando proporcionalmente estudos que contemplam outras faixas etárias, como crianças e idosos. Foram incluídos dez estudos em inglês e um em português, todos publicados em 2020. Os Estados Unidos foram o local de origem da maior parte dos artigos (45,4\%). Os dados dos estudos estão apresentados no Quadro 1.

Em termos clínicos, reconhece-se que a situação de pandemia da COVID-19 apresenta muitas nuances a serem conhecidas. Em relação às doenças respiratórias entre adolescentes ainda não há consenso sobre os riscos, embora os dados coletados com adultos indiquem que a asma é um fator de risco para morbidade e mortalidade da COVID-19 12. Médicos e famílias devem garantir o controle da asma diante das incertezas sobre esse quadro e sua relação com a COVID-19 12. Os adolescentes asmáticos também devem manter o uso da medicação prescrita e aumentar a higienização das mãos e o distanciamento social 12 .

Um dos estudos revisados apresentou o estado da arte sobre aspectos clínicos, diagnósticos, manejo terapêutico e prognóstico entre crianças e adolescentes ${ }^{13}$. Os autores verificaram que a maioria dos pacientes apresentava sintomas clínicos, principalmente febre e tosse 13 . Apenas um caso de infecção grave necessitou de ventilação assistida 13. Em geral, o prognóstico foi bom em todos os estudos e registrou-se apenas um óbito na faixa etária de 10 a 19 anos 13.

Outro estudo, revisando o caso de dois adolescentes com celulite orbitária (infeção dos tecidos moles que envolvem a estrutura orbital), sinusite e infecção por SARS-CoV-2, indicou a associação temporal e a ausência de sintomas crônicos nos dois adolescentes avaliados 14 . Os autores indicam a possibilidade da congestão respiratória secundária provocada pela COVID-19 ter contribuído para agravar a celulite orbitária, chegando em um dos casos à disseminação intracraniana com subsequente abscesso epidural 14 .

A saúde mental é determinada por múltiplos fatores e a situação de pandemia da COVID-19 também a afeta. Nesse sentido, um estudo epidemiológico transversal chinês indicou uma elevada prevalência de sintomas depressivos $(43,7 \%)$ e de ansiedade (37,4\%), bem como uma combinação entre estes sintomas (31,3\%), associada à pandemia, na população geral 15. O sexo feminino foi o maior fator de risco para a sintomatologia depressiva ou de ansiedade. A conscientização sobre a COVID-19 - acesso a informações, conhecimento sobre o processo de adoecimento e as medidas de prevenção - foi considerada como fator de proteção contra a sintomatologia depressiva ou de ansiedade 15 .

Outro estudo conduzido com uma amostra de 584 adolescentes e adultos jovens chineses, duas semanas após a OMS declarar o estado de pandemia pela COVID-19, revelou que 40,4\% estavam propensos a apresentar problemas psicológicos e 14,4\% já manifestavam sintomas de transtorno de estresse pós-traumático ${ }^{16}$. Segundo os autores, o fato de a COVID-19 ter sido declarada pandemia, ocorrer um aumento das informações sobre a doença e uma sobrecarga de notícias sobre casos de contaminação e óbito influenciaram a saúde mental dos participantes 16 .

A situação pandêmica pode ainda provocar irritabilidade e medo de que os membros da família possam se contaminar. Além disso, o caso das mudanças provocadas pela puberdade e a situação de distanciamento social (fechamento das escolas e nenhuma atividade de interação com pares, por exemplo) fazem com que os adolescentes avaliem negativamente seu status no grupo e nos relacionamentos interpessoais 8 . Esses dois aspectos são essenciais na adolescência pois provocam um movimento de afastamento da família e aproximação com os pares, mas que com a pandemia 
Quadro 1

Características, objetivos e principais resultados dos estudos revisados.

\begin{tabular}{|c|c|c|c|c|c|c|}
\hline $\begin{array}{l}\text { Referência } \\
\text { (ano) }\end{array}$ & Periódico & País & Delineamento & $\begin{array}{l}\text { População/ } \\
\text { Amostra }\end{array}$ & Objetivo & Principais resultados \\
\hline $\begin{array}{l}\text { Zhow et al. } 15 \\
(2020)\end{array}$ & $\begin{array}{l}\text { European } \\
\text { Child \& } \\
\text { Adolescent } \\
\text { Psychiatry }\end{array}$ & China & Transversal & $\begin{array}{c}8.079 \\
\text { adolescentes }\end{array}$ & $\begin{array}{l}\text { Avaliar a taxa de } \\
\text { prevalência e correlatos } \\
\text { sociodemográficos dos } \\
\text { sintomas depressivos } \\
\text { e de ansiedade em } \\
\text { adolescentes chineses } \\
\text { afetados pela COVID-19. }\end{array}$ & $\begin{array}{l}\text { Verificou-se alta prevalência } \\
\text { de sintomas depressivos e de } \\
\text { ansiedade nos adolescentes. O sexo } \\
\text { feminino foi o maior fator de risco } \\
\text { para a sintomatologia. Problemas } \\
\text { psicológicos de saúde foram } \\
\text { associados negativamente ao nível } \\
\text { de consciência sobre a COVID-19. }\end{array}$ \\
\hline $\begin{array}{l}\text { Abrams \& } \\
\text { Szefer } 12 \\
(2020)\end{array}$ & $\begin{array}{l}\text { Journal of } \\
\text { Pediatrics }\end{array}$ & $\begin{array}{l}\text { Estados } \\
\text { Unidos }\end{array}$ & Teórico & - & $\begin{array}{c}\text { Fornecer uma visão } \\
\text { geral sobre a COVID-19 } \\
\text { e asma pediátrica. }\end{array}$ & $\begin{array}{l}\text { Ainda não é possível afirmar que a } \\
\text { asma seria uma condição clínica que } \\
\text { favorece morbidade e mortalidade } \\
\text { por COVID-19 na adolescência. }\end{array}$ \\
\hline $\begin{array}{l}\text { Barney et } \\
\text { al. } 18 \text { (2020) }\end{array}$ & $\begin{array}{c}\text { Journal of } \\
\text { Adolescent } \\
\text { Health }\end{array}$ & $\begin{array}{l}\text { Estados } \\
\text { Unidos }\end{array}$ & $\begin{array}{c}\text { Relato de } \\
\text { experiência }\end{array}$ & - & $\begin{array}{l}\text { Descrever a transição } \\
\text { de um serviço } \\
\text { presencial para serviço } \\
\text { de telemedicina. }\end{array}$ & $\begin{array}{l}\text { Descrição da implantação de um } \\
\text { serviço médico de atendimento } \\
\text { virtual. Verificou-se que questões de } \\
\text { saúde geral, saúde mental, saúdes } \\
\text { sexuais e reprodutivas poderiam ser } \\
\text { gerenciadas pela telemedicina. }\end{array}$ \\
\hline $\begin{array}{l}\text { Castagnoli et } \\
\text { al. } 13 \text { 2020) }\end{array}$ & $\begin{array}{c}\text { JAMA } \\
\text { Pediatrics }\end{array}$ & Itália & $\begin{array}{c}\text { Revisão } \\
\text { sistemática }\end{array}$ & 18 artigos & $\begin{array}{c}\text { Avaliar casos } \\
\text { pediátricos (crianças e } \\
\text { adolescentes) relatados } \\
\text { de infecção por } \\
\text { SARS-CoV-2. }\end{array}$ & $\begin{array}{l}\text { Febre e tosse foram os principais } \\
\text { sintomas de crianças e adolescentes } \\
\text { diagnosticados com a COVID-19. } \\
\text { Apenas um caso necessitou de } \\
\text { ventilação assistida e um óbito } \\
\text { na faixa etária de } 10 \text { a } 19 \text { anos foi } \\
\text { documentado. }\end{array}$ \\
\hline $\begin{array}{l}\text { Turbin et } \\
\text { al. } 14 \text { (2020) }\end{array}$ & Orbit & $\begin{array}{l}\text { Estados } \\
\text { Unidos }\end{array}$ & Estudo de caso & 2 casos & $\begin{array}{l}\text { Apresentar dois casos } \\
\text { de adolescentes com } \\
\text { celulite orbitária, } \\
\text { sinusite e infecção por } \\
\text { SARS-CoV-2. }\end{array}$ & $\begin{array}{l}\text { A associação temporal e a ausência } \\
\text { de sintomas crônicos nos dois } \\
\text { adolescentes avaliados indicam } \\
\text { a possibilidade da COVID-19 ter } \\
\text { agravado a celulite orbitária. }\end{array}$ \\
\hline $\begin{array}{l}\text { Hightow- } \\
\text { Weidman et } \\
\text { al. } 20 \text { (2020) }\end{array}$ & $\begin{array}{l}\text { AIDS and } \\
\text { Behavior }\end{array}$ & $\begin{array}{l}\text { Estados } \\
\text { Unidos }\end{array}$ & Teórico & $\begin{array}{l}7 \text { propostas de } \\
\text { ensaios clínicos }\end{array}$ & $\begin{array}{l}\text { Discutir alterações } \\
\text { provocadas pela } \\
\text { COVID-19 em estudos } \\
\text { conduzidos pela } \\
\text { Adolescent Trials } \\
\text { Network for HIV } \\
\text { Interventions. }\end{array}$ & $\begin{array}{l}\text { Estudos e intervenções com jovens } \\
\text { em risco ou vivendo com HIV foram } \\
\text { afetados pela atual situação de } \\
\text { pandemia da COVID-19. }\end{array}$ \\
\hline $\begin{array}{l}\text { Liang et al. } 16 \\
(2020)\end{array}$ & $\begin{array}{l}\text { Psychiatric } \\
\text { Quarterly }\end{array}$ & China & Transversal & $\begin{array}{c}584 \text { adolescentes } \\
\text { e adultos jovens }\end{array}$ & $\begin{array}{l}\text { Avaliar a condição } \\
\text { de saúde mental de } \\
\text { adolescentes e adultos } \\
\text { jovens duas semanas } \\
\text { após o anúncio da } \\
\text { pandemia COVID-19 } \\
\text { pela Organização } \\
\text { Mundial da Saúde. }\end{array}$ & $\begin{array}{c}\text { Cerca de } 40,4 \% \text { dos participantes } \\
\text { estavam propensos a problemas } \\
\text { psicológicos e } 14,4 \% \text { apresentaram } \\
\text { sintomas de transtorno de estresse } \\
\text { pós-traumático. Inferiu-se que } \\
\text { doenças infecciosas como a } \\
\text { COVID-19 podem influenciar na } \\
\text { saúde mental dos adolescentes e } \\
\text { adultos jovens. }\end{array}$ \\
\hline
\end{tabular}

(continua) 


\begin{tabular}{|c|c|c|c|c|c|c|}
\hline $\begin{array}{l}\text { Referência } \\
\text { (ano) }\end{array}$ & Periódico & País & Delineamento & $\begin{array}{c}\text { População/ } \\
\text { Amostra }\end{array}$ & Objetivo & Principais resultados \\
\hline $\begin{array}{l}\text { Imran et al. } 8 \\
\text { (2020) }\end{array}$ & $\begin{array}{c}\text { Pakistan } \\
\text { Journal of } \\
\text { Medical } \\
\text { Sciences }\end{array}$ & Paquistão & Teórico & - & $\begin{array}{c}\text { Analisar a } \\
\text { vulnerabilidade, em } \\
\text { termos de saúde } \\
\text { mental, de crianças } \\
\text { e adolescentes na } \\
\text { situação de pandemia } \\
\text { pela COVID-19. }\end{array}$ & $\begin{array}{l}\text { Verifica-se um impacto no } \\
\text { desenvolvimento e nos processos } \\
\text { de identificação dos adolescentes } \\
\text { que dependem, neste momento } \\
\text { do ciclo vital, dos grupos de pares } \\
\text { em oposição à aproximação com } \\
\text { a família que é favorecida pelo } \\
\text { distanciamento social. }\end{array}$ \\
\hline $\begin{array}{l}\text { Oosterhoff et } \\
\text { al. } 17 \text { (2020) }\end{array}$ & $\begin{array}{c}\text { Journal of } \\
\text { Adolescent } \\
\text { Health }\end{array}$ & $\begin{array}{l}\text { Estados } \\
\text { Unidos }\end{array}$ & Transversal & 683 adolescentes & $\begin{array}{c}\text { Examinar as } \\
\text { motivações, o } \\
\text { envolvimento e a } \\
\text { saúde psicossocial dos } \\
\text { adolescentes para o } \\
\text { distanciamento social. }\end{array}$ & $\begin{array}{l}\text { Os adolescentes demonstraram } \\
\text { envolvimento relativo com } \\
\text { o distanciamento social e as } \\
\text { motivações para tanto estavam } \\
\text { relacionadas à responsabilidade } \\
\text { social e à preocupação com o } \\
\text { adoecimento dos outros. }\end{array}$ \\
\hline $\begin{array}{l}\text { Carlotti et al. } \\
19 \text { (2020) }\end{array}$ & Clinics & Brasil & $\begin{array}{c}\text { Revisão } \\
\text { narrativa }\end{array}$ & - & $\begin{array}{l}\text { Verificar aspectos } \\
\text { epidemiológicos, } \\
\text { clínicos, laboratoriais } \\
\text { e terapêuticos da } \\
\text { COVID-19 em pacientes } \\
\text { pediátricos críticos - } \\
\text { incluindo adolescentes. }\end{array}$ & $\begin{array}{l}\text { Foram priorizados os cuidados } \\
\text { intensivos para produzir um } \\
\text { relatório com o objetivo de } \\
\text { padronizar o atendimento } \\
\text { a crianças e adolescentes } \\
\text { gravemente enfermos. Apresenta- } \\
\text { se um fluxograma para orientar os } \\
\text { profissionais de saúde na triagem } \\
\text { de casos críticos. }\end{array}$ \\
\hline $\begin{array}{l}\text { Marques et } \\
\text { al. } 7 \text { (2020) }\end{array}$ & $\begin{array}{l}\text { Cadernos } \\
\text { de Saúde } \\
\text { Pública }\end{array}$ & Brasil & Teórico & - & $\begin{array}{c}\text { Apresentar um } \\
\text { panorama sobre } \\
\text { a violência contra } \\
\text { mulheres, crianças } \\
\text { e adolescentes em } \\
\text { tempos de pandemia } \\
\text { pela COVID-19. }\end{array}$ & $\begin{array}{c}\text { As medidas sanitárias adotadas } \\
\text { para o controle da contaminação } \\
\text { pelo coronavírus aumentam a } \\
\text { vulnerabilidade dos adolescentes } \\
\text { para sofrerem violência no contexto } \\
\text { doméstico. }\end{array}$ \\
\hline
\end{tabular}

podem provocar frustração, irritação, desconexão emocional, nostalgia e tédio por causa do distanciamento social 8 .

Sobre a medida de distanciamento social, especificamente, uma pesquisa transversal revelou que os adolescentes apresentam uma tendência para aderir à medida 17 . Essa tendência foi justificada por razões pessoais (medo de adoecer) e pró-sociais, incluindo o reconhecimento de que o distanciamento social é uma responsabilidade coletiva e que ajuda a garantir que outras pessoas não fiquem doentes 17. Nesse estudo, quando o distanciamento social ocorreu para impedir a contaminação pessoal ou para evitar julgamentos sociais, houve mais relatos de ansiedade. Em casos em que os amigos solicitaram o afastamento também houve registro de sintomas depressivos 17.

Por outro lado, a vulnerabilidade a situações de violência doméstica no contexto familiar também pode aumentar durante o período de distanciamento social. A partir do momento que as escolas foram fechadas, os adolescentes puderam ficar mais próximos de figuras parentais abusivas que utilizam castigos físicos ou práticas de punição física para controlar comportamentos indesejados 7 . Além 
disso, os adolescentes também podem ficar mais irritadiços devido às restrições de mobilidade e a impossibilidade de estar com os colegas. Essa realidade pode resultar em comportamentos agressivos ou de desobediência, e no aumento da convivência virtual com amigos por meio de tecnologias digitais 7 . A convivência familiar também pode aumentar as tensões nas relações interpessoais e favorecer o surgimento de doenças mentais preexistentes ${ }^{7}$.

As medidas sanitárias para o controle da contaminação pelo SARS-CoV-2 alteraram a rotina de serviços de saúde, como ocorreu na Clínica de Medicina do Adolescente e do Adulto Jovem da Universidade da Califórnia em São Francisco (Estados Unidos). Pesquisadores relataram o rápido processo de transição do atendimento presencial para o atendimento virtual ${ }^{18}$. Foram descritos os passos seguidos pela equipe e cada profissional, e os desafios mais prevalentes foram as resistências ou desconfortos dos usuários adolescentes em aderirem à nova proposta terapêutica 18 . Os problemas gerais de saúde dos adolescentes mais fáceis de serem abordados pela telemedicina incluíram acompanhamento de dores de cabeça crônicas, problemas dermatológicos e algumas queixas musculoesqueléticas 18. Acompanhamento de transtornos do humor, manutenção de medicamentos para o transtorno de déficit de atenção e hiperatividade, orientação relacionada à saúde sexual e reprodutiva e monitoramento de casos de transtornos alimentares também foram ações percebidas como facilmente gerenciadas via telemedicina 18 .

Ainda sobre a lógica dos protocolos de atendimento, um estudo nacional procurou apresentar uma padronização para o atendimento de crianças e adolescentes gravemente enfermos pela COVID-19 19. Os pesquisadores coletaram informações epidemiológicas e os critérios para o diagnóstico diferencial a fim de auxiliar os profissionais na tomada de decisão diante de casos graves que possam aparecer nos serviços de saúde 19. Segundo os pesquisadores, é importante pensar no manejo terapêutico nos serviços de saúde, mas também orientar pais e responsáveis sobre a necessidade de aguardar transporte médico adequado quando a situação está agravada por complicações pela doença 19.

O desenvolvimento de pesquisas também tem sido afetado pela pandemia da COVID-19. Nesse sentido, um dos estudos revisados avaliou uma rede de pesquisa com adolescentes e jovens em risco ou que vivem com o HIV, e revelou que foram necessárias mudanças na implementação de intervenções e perguntas sobre COVID-19 adicionadas aos protocolos de pesquisa 20. Essas ações foram avaliadas como capazes de aumentar a compreensão sobre como a pandemia afeta aqueles em risco e vivendo com HIV, por exemplo 20.

Em síntese, os principais dados extraídos dos 11 artigos revisados sobre a saúde do adolescente fazem referência, principalmente, à saúde mental, a aspectos do desenvolvimento humano e também aos fatores clínicos relacionados à COVID-19. Esses achados estão sintetizados na Figura 2.

As principais limitações assinaladas pelos estudos revisados se referiam ao desenho do tipo transversal das pesquisas 16,17, ao uso de questionários de autorrelato 15,16,17 e a amostras relativamente pequenas ou suas características 15,16,17. O estudo de revisão sistemática ressaltou que as limitações faziam referência ao curto período de publicação, à simplicidade dos estudos revisados e à inclusão apenas de estudos desenvolvidos na China 13. Pesquisas adicionais utilizando delineamento longitudinal ou aumento dos tamanhos amostrais, bem como desenvolvidas em diferentes contextos e com fontes múltiplas de informação foram sugeridas.

\section{Discussão}

Este estudo objetivou identificar o impacto ou os efeitos da pandemia da COVID-19 na saúde dos adolescentes. Nesse sentido, doenças respiratórias, como a asma, merecem atenção na medida em que podem ser fator de risco para a infecção pelo SARS-CoV-2. Verificou-se também problemas de saúde mental dos adolescentes relacionados à doença e às medidas sanitárias adotadas para controlar a contaminação. Especificamente, as medidas de distanciamento social e o fechamento das escolas têm sido vivenciadas de forma negativa. Essas medidas também podem favorecer a ocorrência de violências ou comportamentos agressivos no contexto doméstico. Serviços de saúde que atendem à população adolescente também precisaram reorientar as práticas de cuidado e mesmo pesquisas precisaram ser repensadas. 


\section{Figura 2}

Síntese dos principais resultados dos estudos revisados.

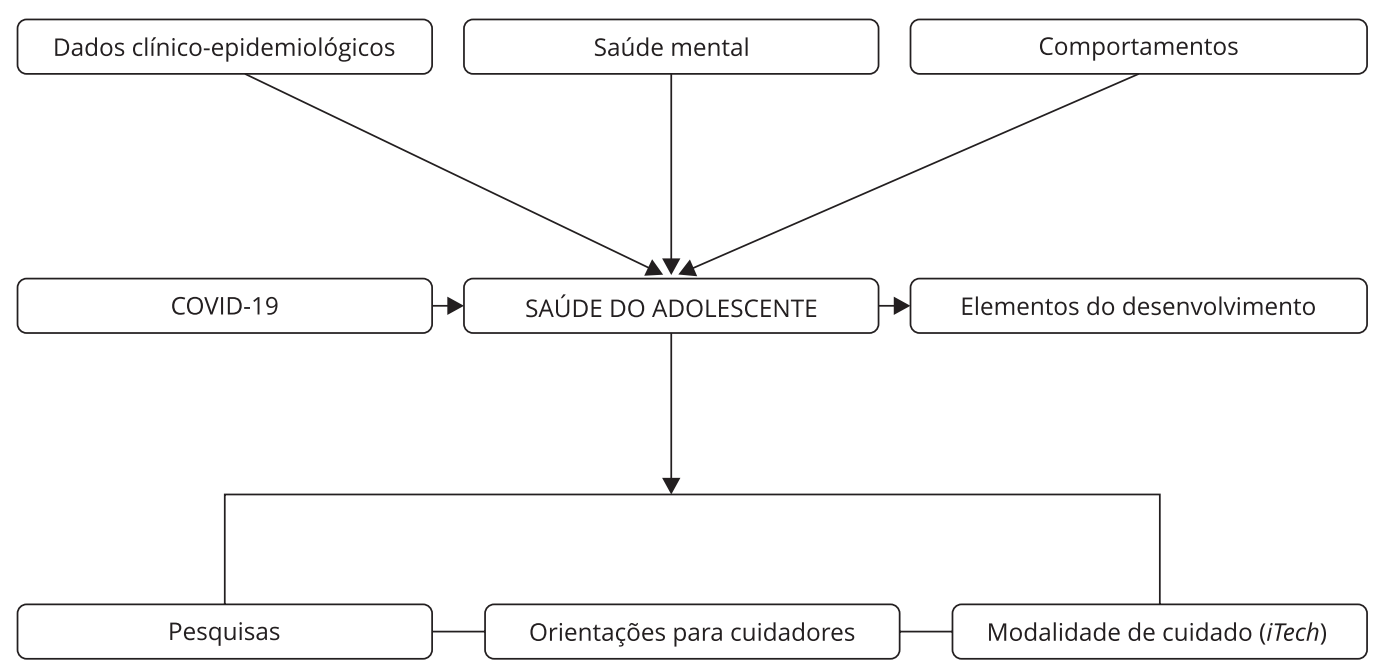

No Brasil, é alta a prevalência de asma em adolescentes. Um estudo nacional, que contou com a participação de 74.589 adolescentes, identificou uma prevalência de 13,1\% da amostra 21. A Pesquisa Nacional de Saúde do Escolar (PeNSE), em sua edição de 2015, reuniu dados de 102.072 escolares do 9o ano do Ensino Fundamental de todos os estados brasileiros e do Distrito Federal, registrou que 17,92\% dos estudantes tinham relato de episódio de asma alguma vez na vida 22. Casos de chiado no peito também foram registrados como sintoma comum $(23,52 \%)$ entre adolescentes 22 . Esses resultados sinalizam a importância de profissionais e equipes de saúde acompanharem os casos de adolescentes com doenças respiratórias já notificadas e que podem estar em risco aumentado para a COVID-19.

Além disso, com a pandemia já se documentou o aumento do risco para o desenvolvimento de quadros de ansiedade, depressão e outros problemas psicológicos. Isso ocorre devido ao aumento do estresse referente às características da própria pandemia, à diminuição da mobilidade, à desaceleração econômica e ao fechamento das escolas, que são as medidas amplamente adotadas para controlar o contágio pelo novo coronavírus e impedir o colapso dos sistemas de saúde ${ }^{5}$. Diante desse cenário, as pessoas tendem a ficar mais ansiosas, com humor deprimido e podem adotar comportamentos extremos de risco à saúde, como ideação ou tentativa de suicídio e automutilação 23.

Esse tipo de interpretação a respeito do agravamento das condições de saúde mental, decorre de outros momentos de pandemia ou catástrofe da história recente da humanidade. Mesmo considerando que são períodos diversos, o estudo sobre esses momentos pode sinalizar o que ocorre e como agir no pós-pandemia da COVID-19. Por exemplo, durante o surto da SARS em 2003 foram relatados sintomas psiquiátricos de intensidade leve a grave, além de quadros de ansiedade, solidão, tédio, raiva, preocupação com os efeitos da quarentena e medo do contágio dos membros da família e amigos 24. Em outra perspectiva, entre dois ou três anos após os ataques de 11 de setembro de 2001 nos Estados Unidos, descobriu-se que os adolescentes, entre outros grupos, foram os mais afetados emocionalmente 25 .

Essas experiências de adversidade relacionadas a situações limites se estendem na história pessoal dos indivíduos e pressupõe-se que, no caso da pandemia provocada pelo coronavírus em 2020, repercutirá emocional e psicologicamente a longo prazo 24 . Para os adolescentes, além da situação pandêmica, há o distanciamento social e a interrupção da rotina de vida diária que inclui a frequência à escola e o encontro com o grupo de pares. Em termos de desenvolvimento, a adolescência é um momento 
de maior distanciamento das figuras parentais e família e maior aproximação com os colegas, o que em períodos de pandemia não é possível diante da necessidade de ficar em casa ${ }^{8}$. Nota-se ainda que o distanciamento social pode mobilizar mudanças internas ou questões subjacentes à pandemia que precipitam o surgimento de quadros psiquiátricos.

Nesse sentido, problemas psicológicos e de saúde mental são temas frequentes na literatura sobre a saúde do adolescente. Em geral, já foi documentada uma prevalência de 30\% de transtornos mentais comuns em adolescentes brasileiros 26 . Aqueles mais comuns são caracterizados principalmente por sintomas ligados à depressão, ansiedade, queixas inespecíficas e somatização. As famílias podem desencadear processos de crise, principalmente quando a dinâmica familiar é marcada por violências 27 , o que pode ser ampliado durante as situações de distanciamento social. Por outro lado, quando as interações familiares são confiáveis, pautadas no suporte emocional e social 27, elas podem ser fatores protetivos para a saúde mental, modelo que deve ser trabalhado nas iniciativas de cuidado às famílias.

Serviços de saúde direcionados para a população adolescente também devem considerar essas questões, mesmo diante da impossibilidade de propiciar atendimentos presenciais. Os estudos revisados sinalizaram como a pandemia afetou a rotina dos serviços e a necessidade de implantação rápida de modelos complementares para não interromper os atendimentos. A possibilidade do atendimento on-line ou da telemedicina é uma tendência válida principalmente entre os adolescentes que são familiarizados com o uso das tecnologias e de diferentes mídias, favorecendo a adesão às intervenções digitais de saúde, incluindo aquelas que abordam os comportamentos de prevenção e assistência em si 20. Contudo, já foram documentadas reações negativas e agressivas diante da mudança na modalidade de atendimento - do presencial para o virtual 28.

Em outra direção, o maior tempo no ambiente doméstico também pode favorecer o aumento das situações de violência contra adolescentes. Essa análise emerge de estudos que estão sendo iniciados sobre a violência doméstica e que incluem os adolescentes nos grupos de risco, além das mulheres e das crianças 7. A violência doméstica contra os adolescentes também é considerada um problema de saúde pública ${ }^{29}$. Ela é caracterizada pelo uso de práticas de punição física para disciplinar ou corrigir comportamentos indesejados, pelas agressões físicas, psicológicas, sexuais e casos de negligência parental 30 . Como fenômeno social, a violência doméstica é entendida como resultado de processos dinâmicos e psicossociais baseados na interação familiar ${ }^{29}$. Nesse sentido, com o distanciamento social adotado no momento da pandemia, pais e responsáveis abusadores podem aumentar as agressões.

Os adolescentes também podem apresentar comportamentos agressivos ou de delinquência durante o período em que estão em casa com suas famílias. A adolescência é um momento do desenvolvimento marcado pelas mudanças psicobiológicas que podem provocar um aumento da instabilidade emocional e maior irritabilidade 31 . Também ocorre nesse momento o processo de definição da identidade que depende, sobremaneira, da relação com os pares 32. Na pandemia da COVID-19 os encontros com os colegas estão diminuídos ou inexistem e isto pode aumentar o estresse, desencadeando comportamentos negativos/indisciplinados ou aumento do uso de tecnologias digitais. Pesquisas futuras são necessárias para examinar as facetas e o impacto do momento de distanciamento social para os adolescentes.

Pais e responsáveis podem auxiliar os adolescentes neste momento, ajudando-os a compreender de forma cognitiva ou emocional a importância das medidas sanitárias que estão sendo adotadas para o controle da COVID-19. Entretanto, muitos deles não sabem como fazer isso. Explicar aspectos da curva de contágio e o seu efeito na saúde pública é uma alternativa de abordagem cognitiva. Emocionalmente, os adolescentes podem ser sensibilizados sobre o cuidado consigo, que pode resultar na não contaminação de outras pessoas próximas ou mesmo estranhas. Um dos estudos revisados confirmou que os adolescentes têm disponibilidade interna para aderir e compreender a importância do distanciamento social ${ }^{17}$. Os adolescentes também devem ser incluídos nas atividades domésticas, pois isto restituí o senso de autoeficácia, caracterizada pela avaliação de que se é capaz de fazer com sucesso alguma coisa em momentos de ruptura com o cotidiano escolar ou social.

Por fim, embora as evidências apresentadas sejam importantes, elas devem ser interpretadas considerando suas três principais limitações. Inicialmente, o nível de evidência dos estudos revisados não foi mensurado, conforme pressupõem as diretrizes para o desenvolvimento de scoping 
review. Em segundo lugar, as pesquisas sobre o impacto da pandemia da COVID-19 na adolescência são em número reduzido, e muitas informações já documentadas são obtidas valendo-se de hipóteses baseadas em outras experiências de adversidade. Terceiro, o critério de inclusão relacionado ao idioma de publicação também pode ser considerado uma limitação, pois como foi na China que desencadeou toda a pandemia, estudos publicados apenas em mandarim podem ter sido ignorados ou não localizados.

\section{Considerações finais}

De forma exploratória, esta scoping review objetivou identificar evidências científicas sobre o impacto da questão emergente de pandemia da COVID-19 na adolescência. Verificou-se que as vivências dos adolescentes na pandemia da COVID-19 ainda são pouco exploradas por estudos e existem diversas lacunas no conhecimento. Os adolescentes apresentam os mesmos sintomas clínicos que os adultos quando infectados pelo novo coronavírus. Problemas de saúde mental e violência doméstica neste momento do desenvolvimento foram associados à pandemia ou ao distanciamento social que, em alguma medida, é compreendida e aceita pelos adolescentes. A contribuição original do estudo reside na abordagem da saúde do adolescente em tempos de pandemia. Nesse sentido, considerando a definição ampliada do período da adolescência, percebe-se a necessidade de estudos com capacidade de avaliar as características societárias que devem influenciar a saúde individual e coletivamente dos adolescentes e seus familiares que coabitam durante o distanciamento social.

Pesquisas epidemiológicas com a população adolescente são recomendadas, assim como estudos capazes de capturar nuances das experiências subjetivas dos adolescentes diante um problema que causa medo, incertezas e insegurança. Em termos de atuação e boas práticas de cuidado, recomenda-se que equipes de saúde na atenção primária desenvolvam ações de educação e promoção de saúde junto aos adolescentes, mesmo que virtualmente, pois verificou-se boa adesão deste público às modalidades complementares de cuidado. Essas visam a esclarecer os adolescentes sobre as medidas sanitárias adotadas para o controle da contaminação ou ainda estimular o desenvolvimento da responsabilidade social que pressupõe o autocuidado e o cuidado com o outro. 


\section{Colaboradores}

W. A. Oliveira concebeu e desenhou o estudo, contribuiu na coleta e interpretação dos dados e escreveu o manuscrito. J. L. Silva contribuiu na coleta, análise, interpretação dos dados, revisão do manuscrito e aprovou a versão final. A. L. M. Andrade, D. De Micheli e D. M. Carlos contribuíram na análise e interpretação dos dados, revisão do manuscrito e aprovou a versão final. M. A. I. Silva concebeu o estudo, contribuiu na análise e interpretação dos dados, revisão do manuscrito e aprovou a versão final.

\section{Informações adicionais}

ORCID: Wanderlei Abadio de Oliveira (0000-00023146-8197); Jorge Luiz da Silva (0000-0002-37278490); André Luiz Monezi Andrade (0000-00030111-8935); Denise De Micheli (0000-0001-85464354); Diene Monique Carlos (0000-0002-49507350); Marta Angélica Iossi Silva (0000-0002-99678158).

\section{Agradecimentos}

Os autores agradecem o apoio do Conselho Nacional de Desenvolvimento Científico e Tecnológico $(\mathrm{CNPq})$ oferecido por meio de bolsas de produtividade para D. De Micheli e M. A. I. Silva (processos: 304684/2017-1 e 305469/2019-3, respectivamente).

\section{Referências}

1. Sawyer SM, Azzopardi PS, Wickremarathne D, Patton GC. The age of adolescence. Lancet Child Adolesc Health 2018; 2:223-8.

2. Schaefer R, Barbiani R, Nora CRD, Viegas K, Leal SMC, Lora PS, et al. Políticas de saúde de adolescentes e jovens no contexto luso-brasileiro: especificidades e aproximações. Ciênc Saúde Colet 2018; 23:2849-58.

3. Vinagre MG, Barros L. Preferências dos adolescentes sobre os cuidados de saúde. Ciênc Saúde Colet 2019; 24:1627-36.

4. Vilelas JMS. O novo coronavírus e o risco para a saúde das crianças. Rev Latinoam Enferm 2020; 28:e3320.

5. Oliveira WA, Oliveira-Cardoso EA, Silva JL, Santos MA. Impactos psicológicos e ocupacionais das sucessivas ondas recentes de pandemias em profissionais da saúde: revisão integrativa e lições aprendidas. Estud Psicol (Campinas) 2020; 37:e200066.

6. Enumo SRF, Weide JN, Vicentini ECC, Araujo MF, Machado WL. Enfrentando o estresse em tempos de pandemia: proposição de uma cartilha. Estud Psicol (Campinas) 2020; 37:e200065.

7. Marques ES, Moraes CL, Hasselmann MH, Deslandes SF, Reichenheim ME. A violência contra mulheres, crianças e adolescentes em tempos de pandemia pela COVID-19: panorama, motivações e formas de enfrentamento. Cad Saúde Pública 2020; 36:e00074420.

8. Imran N, Zeshan M, Pervaiz Z. Mental health considerations for children \& adolescents in COVID-19 pandemic. Pak J Med Sci 2020; 36(COVID19-S4):S67-72.

9. Bruns DP, Kraguljac NV, Bruns TR. COVID-19: facts, cultural considerations, and risk of stigmatization. J Transcult Nurs 2020; $31: 326-32$

10. Joanna Briggs Institute. The Joanna Briggs Institute reviewers' manual 2015: methodology for JBI scoping reviews. Adelaide: Joanna Briggs Institute; 2015.

11. Moher D, Liberati A, Tetzlaff J, Altman DG; PRISMA Group. Preferred reporting items for systematic reviews and meta-analyses: the PRISMA statement. PLoS Med 2009; 6:e1000097.

12. Abrams EM, Szefler SJ. Managing asthma during coronavirus disease-2019: an example for other chronic conditions in children and adolescents. J Pediatr 2020; 222:221-6.

13. Castagnoli R, Votto M, Licari A, Brambilla I, Bruno R, Perlini S, et al. Severe acute respiratory syndrome coronavirus 2 (SARS-CoV-2) infection in children and adolescents: a systematic review. JAMA Pediatr 2020; [Epub ahead of print].

14. Turbin RE, Wawrzusin PJ, Sakla NM, Traba CM, Wong KG, Mirani N, et al. Orbital cellulitis, sinusitis and intracranial abnormalities in two adolescents with COVID-19. Orbit 2020; 39:305-10 
15. Zhou S-J, Zhang L-G, Wang L-L, Guo Z-C, Wang J-Q, Chen J-C, et al. Prevalence and socio-demographic correlates of psychological health problems in Chinese adolescents during the outbreak of COVID-19. Eur Child Adolesc Psychiatry 2020; 29:749-58.

16. Liang L, Ren H, Cao R, Hu Y, Qin Z, Li C, et al. The effect of COVID-19 on youth mental health. Psychiatr Q 2020; [Epub ahead of print].

17. Oosterhoff B, Palmer CA, Wilson J, Shook N. Adolescents' motivations to engage in social distancing during the COVID-19 pandemic: associations with mental and social health. J Adolesc Health 2020; 67:179-85.

18. Barney A, Buckelew S, Mesheriakova V, Raymond-Flesch M. The COVID-19 pandemic and rapid implementation of adolescent and young adult telemedicine: challenges and opportunities for innovation. J Adolesc Health 2020; 67:164-71.

19. Carlotti APCP, Carvalho WB, Johnston C, Rodriguez IS, Delgado AF. COVID-19 diagnostic and management protocol for pediatric patients. Clinics 2020; 75:e1894.

20. Hightow-Weidman L, Muessig K, Claude K, Roberts J, Zlotorzynska M, Sanchez T. Maximizing digital interventions for youth in the midst of covid-19: lessons from the adolescent trials network for HIV interventions. AIDS Behav 2020; [Epub ahead of print].

21. Kuschnir FC, Gurgel RQ, Solé D, Costa E, Felix MMR, Oliveira CL, et al. ERICA: prevalence of asthma in Brazilian adolescents. Rev Saúde Pública 2016; 50 Suppl 1:13s.

22. Ribeiro-Silva RC, Barreto ML, Ramos D, Cruz AA, Oliveira-Campos M, Malta DC. Asthma trend in adolescence in Brazil: results of the National Adolescent School-based Health Survey (PeNSE 2012-2015). Rev Bras Epidemiol 2018; 21 Suppl 1:e180017.
23. Schmidt B, Crepaldi MA, Bolze SDA, NeivaSilva L, Demenech LM. Saúde mental e intervenções psicológicas diante da pandemia do novo coronavírus (COVID-19). Estud Psicol (Campinas) 2020; 37:e200063.

24. Bahn GH. Coronavirus disease 2019, school closures, and children's mental health. Journal of the Korean Academy of Child and Adolescent Psychiatry 2020; 31:74-9.

25. Mann MJ, Kristjansson AL, Sigfusdottir ID, Smith ML. The role of community, family, peer, and school factors in group bullying: implications for school-based intervention. J School Health 2015; 85:477-86.

26. Lopes CS, Abreu GA, Santos DF, Menezes PR, Carvalho KMB, Cunha CF, et al. ERICA: prevalence of common mental disorders in Brazilian adolescents. Rev Saúde Pública 2016; 50 Suppl 1:14s.

27. Rossi LM, Marcolino TQ, Speranza M, Cid MFB. Crise e saúde mental na adolescência: a história sob a ótica de quem vive. Cad Saúde Pública 2019; 35:e00125018.

28. Fegert JM, Schulze UME. COVID-19 and its impact on child and adolescent psychiatry - a German and personal perspective. Ir J Psychol Med 2020; [Online ahead of print].

29. Barros AS, Freitas MFQ. Violência doméstica contra crianças e adolescentes: consequências e estratégias de prevenção com pais agressores. Pensando Fam 2015; 19:102-14.

30. Quadros MN, Kirchner RM, Hildebrandt LM, Leite MT, Costa MC, Sarzi DM. Situación de la violencia contra niños y adolescentes en Brasil. Enferm Glob 2016; 15:162-73.

31. Senna SRCM, Dessen MA. Reflexões sobre a saúde do adolescente brasileiro. Psicol Saúde Doenças 2015; 16:217-29.

32. Melo MCB, Falbo Neto GH, Alchieri JC, Figueiroa JN. Avaliação do comportamento agressivo de adolescentes. Ciênc Saúde Colet 2015; 20:1861-8. 
Abstract

COVID-19, the disease caused by SARS-CoV-2 (novel coronavirus), emerged in China in December 2019 and spread quickly throughout the world. In this scenario, the current study aimed to identify the impact or effects of the COVID-19 pandemic on adolescents' health. This is a scoping literature review based on the following databases: Web of Science, CINAHL, PsycINFO, SciELO, and PUBCOVID 19. The study adopted the stages proposed by the Joanna Briggs Institute for scoping reviews, and the question orienting the procedures consisted of the acronym PCC (population; concept; context). Eleven articles were included in the review. Clinically, adolescents present the same COVID-19 symptoms as adults. The pandemic and the health measures taken to control transmission were found to be associated with mental health problems in adolescents. Specifically, adolescents have a negative experience with social distancing measures and closing of schools. These measures can also favor situations of violence or aggressive behaviors in the home environment. Healthcare services that treat the adolescent population had to reorient their practices, adopting a virtual model to replace face-to-face care, and even research projects involving adolescence had to be rethought. This scoping review addressed an emerging theme in relation to a population that has received little attention in studies on COVID-19. The results suggest that the pandemic can be considered a determinant that affects different dimensions of adolescents' lives.

COVID-19; Pandemics; Quarentine; Adolescent Health; Health Vulneralibility

\section{Resumen}

La COVID-19, enfermedad provocada por el SARS-CoV-2 (nuevo coronavirus), surgió en China en diciembre de 2019 y se extendió rápidamente por todo el mundo. Ante este escenario, este estudio tuvo como objetivo identificar el impacto o los efectos de la pandemia de la COVID-19 en la salud de los adolescentes. Se trata de una revisión de la literatura del tipo scoping review, valiéndose de las siguientes bases de datos: Web of Science; CINAHL; PsycINFO; SciELO; PUBCOVID 19. El estudio siguió las etapas propuestas por el Instituto Joanna Briggs para las scoping reviews, $y$ el planteamiento que orientó los procedimientos adoptados se construyó a través del acrónimo PCC (población; concepto; contexto). Se incluyeron 11 artículos en la revisión. Clínicamente, los adolescentes presentan los mismos sintomas de la COVID-19 que los adultos afectados por la enfermedad. Se verificó que la pandemia y las medidas sanitarias adoptadas para controlar la infección se asocian a problemas de salud mental en adolescentes. Específicamente, los adolescentes han experimentado de forma negativa las medidas de distanciamiento social y cierre de escuelas. Estas medidas también pueden favorecer la ocurrencia de violencia o comportamientos agresivos en el contexto doméstico. Servicios de salud que atienden a la población adolescente necesitaron reorientar las prácticas de cuidado, adoptando el modelo virtual en sustitución del cuidado presencial, e incluso se necesitaron repensar las investigaciones. Esta scoping review abordó un tema emergente, en relación con una población poco considerada en estudios sobre la COVID-19. Los resultados sugieren que la situación de pandemia puede ser considerada un determinante que afecta diferentes dimensiones de la vida de los adolescentes.

COVID-19; Pandemias; Cuarentena; Salud del Adolescente; Vulnerabilidad en Salud
Recebido em 03/Jun/2020

Versão final reapresentada em 25/Jun/2020

Aprovado em 08/Jul/2020 